\title{
Urate crystals induce macrophage PAF-AH secretion which is differentially regulated by TGFß1 and hydrocortisone
}

\author{
DARSHNA YAGNIK and FRANK HILLS \\ Department of Natural Sciences, Biomarker Research Group, School of Science and Technology, Middlesex University, \\ The Burroughs, London NW4 4BT, UK
}

Received October 9, 2017; Accepted June 13, 2018

DOI: $10.3892 / \mathrm{mmr} .2018 .9323$

\begin{abstract}
The aim of the present study was to establish the role of platelet-activating factor acetyl hydrolase (PAF-AH) in the resolution phase of gout using an established in vitro mononuclear cell model. The effects of signalling pathway inhibitors on PAF-AH secretion, as well as the effects of the common treatments hydrocortisone and colchicine, an antibody against the anti-inflammatory cytokine transforming growth factor $\beta 1$ (TGF $\beta 1$ ), and the transcriptional inhibitor actinomycin $\mathrm{D}$, were also investigated. The effect of recombinant PAF-AH on cytokine secretion by these cells was also determined. Human peripheral blood-derived monocytes were isolated and differentiated into macrophages. Monocytes and macrophages were stimulated with monosodium monohydrate urate (MSU) crystals or lipopolysaccharide in the presence or absence of AEG3482 [a c-Jun N-terminal kinase (JNK) inhibitor], MG132 (a proteasome inhibitor), hydrocortisone or colchicine. Cultures were then analysed for PAF-AH secretion using ELISA. A 6-fold upregulation of PAF-AH secretion was observed following macrophage exposure to MSU crystals for $24 \mathrm{~h}(29.3 \pm 6$ vs. $5.4 \pm 0.3 \mathrm{ng} / \mathrm{ml}$ unstimulated; $\mathrm{P}<0.05)$. Following $72 \mathrm{~h}$, PAF-AH levels decreased significantly $(11.1 \pm 1.8 ; \mathrm{P}<0.01)$. Secretion was further enhanced following pre-treatment with the JNK protein kinase inhibitor AEG3482 prior to MSU crystal stimulation $(\mathrm{P}<0.05)$ and was abrogated when cells were preincubated with actinomycin D or the proteasome inhibitor MG132 (50, 100 and $200 \mu \mathrm{M})$. The addition of recombinant PAF-AH (2.5-10 $\mathrm{ng} / \mathrm{ml})$ to MSU crystal-stimulated immature monocyte cultures significantly decreased pro-inflammatory interleukin (IL)-1 $\beta$ (unstimulated $687 \pm 124$ vs. stimulated $113 \pm 30 \mathrm{pg} / \mathrm{ml}$ ) and IL-6 secretion
\end{abstract}

Correspondence to: Dr Darshna Yagnik, Department of Natural Sciences, Biomarker Research Group, School of Science and Technology, Middlesex University, Room T154, Town Hall Annexe, The Burroughs, London NW4 4BT, UK

E-mail: d.yagnik@mdx.ac.uk

Key words: gout, arthritis, monosodium monohydrate urate crystals, macrophages, platelet activating factor aceytlhydrolase, inflammation, interleukin-6, hydrocortisone, transforming growth factor $\beta 1$, colchicine (unstimulated $590 \pm 50$ vs. stimulated $182 \pm 19 \mathrm{pg} / \mathrm{ml}$ ). Treatment of MSU crystal-stimulated macrophages with hydrocortisone $(2 \mu \mathrm{M})$ also significantly decreased PAF-AH release $(\mathrm{P}<0.05)$. Neutralising anti-TGF $\beta 1$ addition decreased PAF-AH dose-dependently with the highest inhibition observed at $1 \mu \mathrm{g} / \mathrm{ml}(\mathrm{P}<0.05)$. The results implicated that PAF-AH may have an anti-inflammatory role in the resolution phase of gout.

\section{Introduction}

Gout was often referred to as an affliction of kings due to its association with a lavish diet and excess alcohol consumption. In fact, Henry VIII was reported to have suffered from gout (1). The last few decades has seen a global rise in new patient diagnoses (2) and the prevalence of the disease has increased particularly in the UK (3). Gout is an inflammatory form of crystalline arthritis characterised by the deposition of excess circulating uric acid in the form of monosodium monohydrate urate (MSU) crystals into intra-articular joints and tissue spaces (4). Patients experience recurrent acute flares of severe joint inflammation. Such attacks involve inflammation caused by the interaction between MSU crystals and the local tissue environment including intra-articular cells, monocytes and macrophages. The active stage of gout is defined by an inflammatory innate response involving monocytes and neutrophils. This response is driven in part by NLRP3 inflammasome activation via NOD-like receptors which activate interleukin-1 $\beta$ (IL-1 $\beta$ ) release (5-7). Studies in vitro suggest that MSU crystals may induce tumour necrosis factor $\alpha$ (TNF $\alpha$ ), IL-1 $\beta$, IL-6, platelet-activating factor (PAF) secretion in undifferentiated monocytes which in turn may promote endothelial E-selectin expression and secondary neutrophil adhesion (8-10). Conversely, differentiated macrophages are able to uptake MSU crystals and release transforming growth factor $\beta 1$ (TGF $\beta 1$ ), a powerful inhibitor of inflammation (11).

Therapeutic options for gout management remain limited to the use of anti-inflammatory treatments such as non-steroidal anti-inflammatory drugs (NSAIDs), IL-1 $\beta$ antagonists, adrenocorticotrophic hormone (ACTH), colchicine, allopurinol, or hydrocortisone injections following acute flares. A study reviewing patients with tophaceous gout and the risk of complications reported that for all patients there was at least a $25 \%$ casual risk of arterial hypertension, hyperlipidaemia, diabetes, renal function impairment and cardiovascular issues. 
Even after treatment these patients had at least three gout flares per year $(6,7)$. Mononuclear cells are a vital part of innate immunity and have been identified to secrete multiple enzymes into the microenvironment. However, information regarding the role of enzymes secreted in gout afflicted joints is limited. Recently published guidelines by the European League Against Rheumatism have highlighted the potential of enzymes such as febuxostat (xanthine oxidase inhibitor) and pegloticase (acts as an uricase) which target uric acid metabolism, as therapeutic agents in gout $(12,13)$.

Platelet-activating factor acetyl hydrolase (PAF-AH) has multiple roles in numerous pathologies and synthesis and secretion of PAF-AH are increased during monocyte to macrophage differentiation (14-16). Two PAF-AH isoforms have been identified; plasma PAF-AH and intracytoplasmic PAF-AHII. Plasma PAF-AH is a polypeptide with molecular weight $40 \mathrm{kDa}$, mostly produced by macrophages and hepatocytes. It is an anti-inflammatory enzyme since it is predominantly involved in degrading inflammatory phospholipids and is found mainly bound to low density lipoprotein (LDL) in the circulation (17-19). Plasma PAF-AH deficiency has been linked to inflammatory reactions such as sepsis, cardiovascular disease and anaphylaxis $(20,21)$.

PAF-AH resolves inflammation through the inactivation of PAF, a potent, inflammatory lipid mediator. Serum PAF concentrations are rigorously controlled by tight regulation of synthesis and degradation. The acetyl sn-2 group on the backbone of PAF is required for biological activity and is the target for PAF-AH action via esterification converting PAF to its inactive lysosomal-PAF form (22). We have previously shown that immature monocytes pre-treated with recombinant PAF-AH showed a dose-dependent inhibition of TNF $\alpha$ synthesis (12). The mechanism by which PAF-AH is regulated is still not fully understood and so we have investigated the effect of common gout therapeutics on PAF-AH expression. The aim of this study was to investigate the effect of MSU crystal stimulation on macrophage PAF-AH secretion in vitro and to determine the factors and pathways that may be involved in this process.

\section{Materials and methods}

Materials. Hydrocortisone (HC) actin D, lipopolysaccharide (LPS) Dulbecco's modified Eagle medium (DMEM), dimethyl-ethyl-sulphonyl-oxide, HANKS balanced salt solution, histopaque, phosphate buffer saline (PBS), Colchicine and methanol were purchased from Sigma Aldrich, Poole, UK. Proteasome inhibitor (MG132) and c-Jun N-terminal kinase (JNK) inhibitor (AEG3482) were purchased from Tocris Bioscience UK. Cyclic adenosine monophosphate inhibitor (cAMPi) and MSU crystals were purchased from Enzo Life Sciences, Germany. Monoclonal mouse IgG1 clone 9016, mouse anti-human TGF $\beta 1$ (MAB240), recombinant PAF-AH, IL-1 $\beta$ and IL-6 ELISA kits and cell lysis solution were purchased from R\&D Systems, Abingdon, UK. Manufacturer's instructions were followed for all listed kits and reagents. Mouse anti-human IgG1 monoclonal antibody (MAB3832) was used at $100 \mu \mathrm{g} / \mathrm{ml}$, mouse anti-human CD163 IgG1 monoclonal antibody (clone 215927) was used at $2.5 \mu \mathrm{g}$ per $10^{5}$ cells and were both purchased from R\&D Systems, Abingdon, UK. Goat

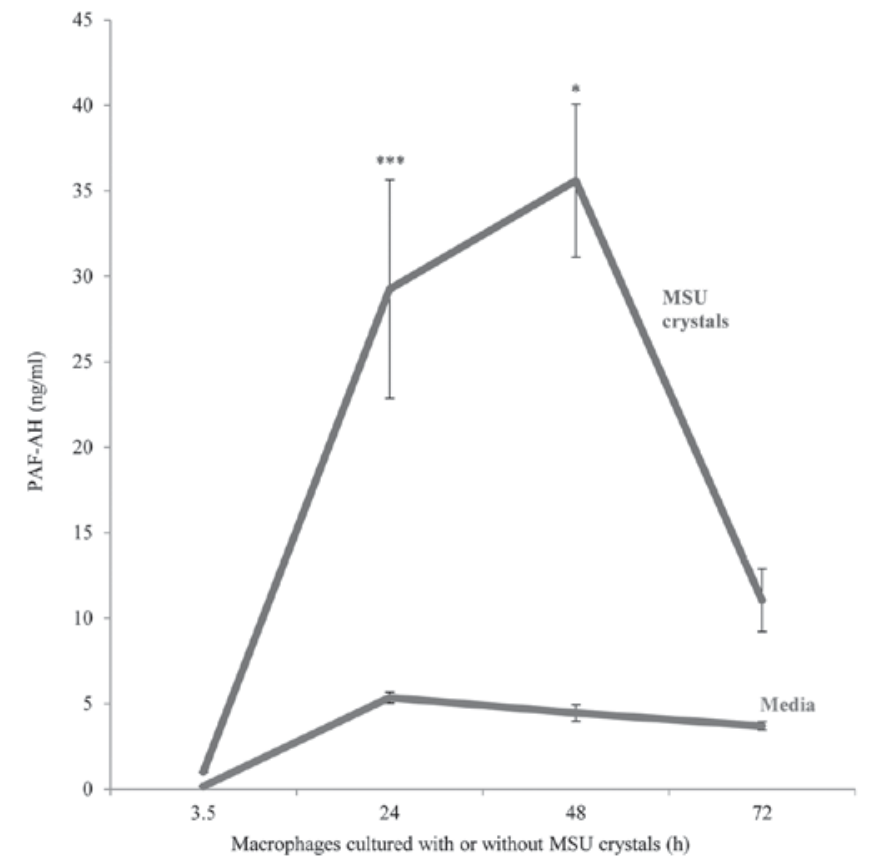

Figure 1. Time course of PAF-AH production. Results are presented as the mean and standard deviation of 3 similar experiments. Peak PAF-AH release was detected at 24-48 $\mathrm{h}$ followed by a decline in enzyme release following $48 \mathrm{~h}$. A significant increase in PAF-AH was detected at $24 \mathrm{~h}$ when compared to $3.5 \mathrm{~h}$ following macrophage MSU crystal stimulation. ${ }^{*} \mathrm{P}<0.05$ and ${ }^{* * *} \mathrm{P}<0.001$ vs. Media. PAF-AH, platelet-activating factor acetyl hydrolase; MSU, monosodium monohydrate urate crystals.

ant-human IgG whole molecule FITC was used at a 1:64 dilution in PBS and purchased from Sigma, Aldrich, Poole, UK.

Monocyte and macrophage preparation. The present study incorporated a series of in vitro cell cultures of differentiated monocytes isolated from human blood cones purchased from the National Health Service Blood Bank (Colindale, London, England). Ethical approval was granted by the Middlesex University Institutional Ethics Committee, Department of Natural Sciences (London, England) and this was also a purchase requirement from the blood bank. The present study complied with the ethical standards established in 1964 in the Declaration of Helsinki.

Leucocyte rich blood cones and blood group AB positive serum were purchased from the NHS Cord blood and transplant bank (Colindale, London, UK). The cones were washed with PBS to and centrifuged on histopaque at $620 \mathrm{x}$ g for $20 \mathrm{mins}$ to harvest leucocytes. The monocyte enriched fraction was obtained from the interface, washed in HANKS balanced solution without calcium or magnesium, after which cells were counted and cultured into 24 well plates at $4 \times 10^{6}$ per $\mathrm{ml}$ in DMEM containing $1 \%$ penicillin and streptomycin at $37^{\circ} \mathrm{C}$ in an atmosphere containing $5 \% \mathrm{CO}_{2}$. The mononuclear cells were allowed to adhere for $1 \mathrm{~h}$ at $37^{\circ} \mathrm{C}$ after which any non-adherent cells were removed by aspiration and washing with PBS. The adherent cells were then cultured in Dulbecco's media containing $10 \% \mathrm{AB}$ serum either for seven days to obtain macrophages or overnight for use as monocytes (9-11). These methods were established previously and macrophage differentiation was determined by light microscopy and flow cytometry phenotypic analysis using the macrophage differentiation 

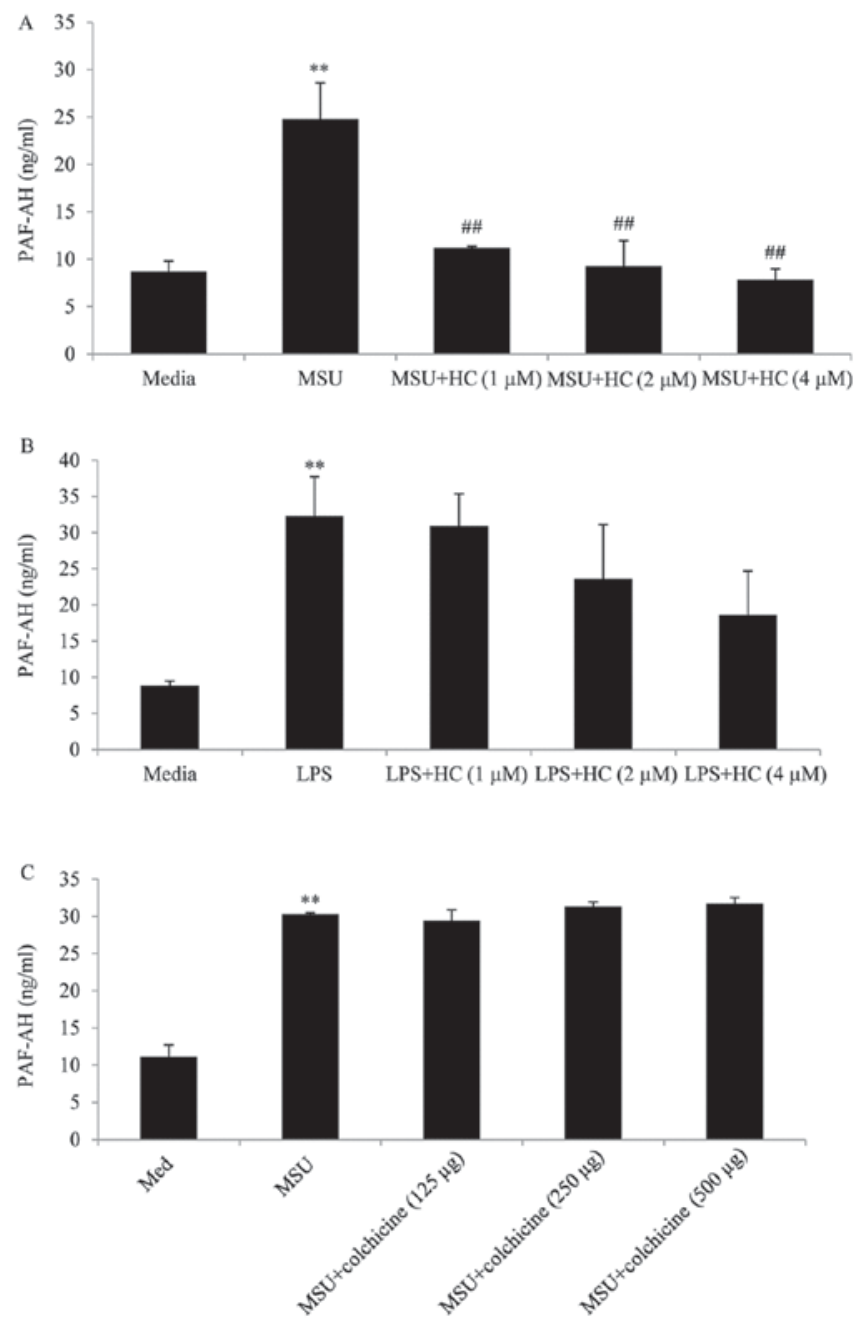

Figure 2. Effect of hydrocortisone and colchicine on PAF-AH secretion by macrophages stimulated with MSU crystals or lipopolysaccharide. Differentiated macrophages were pre-treated with $\mathrm{HC}(1-4 \mu \mathrm{M})$ for $30 \mathrm{~min}$ prior to the addition of (A) $0.5 \mathrm{mg} / \mathrm{ml} \mathrm{MSU}$ crystals or (B) $10 \mathrm{mg} / \mathrm{ml} \mathrm{LPS} \mathrm{and}$ (C) $0.5 \mathrm{mg} / \mathrm{ml} \mathrm{MSU}$ crystals and colchicine for $24 \mathrm{~h}$. Collected supernatants were analysed for PAF-AH by ELISA. Comparisons between macrophages in media only with MSU or LPS stimulation were significantly different Macrophage MSU crystal-but not LPS-stimulated PAF-AH secretion was significantly decreased in the presence of $\mathrm{HC}$ at all doses tested. The results are presented as the mean \pm standard error of the mean. ${ }^{* *} \mathrm{P}<0.01$ vs. Media; ${ }^{\# \#} \mathrm{P}<0.01 \mathrm{vs}$. MSU alone. PAF-AH, platelet-activating factor acetyl hydrolase; $\mathrm{HC}$, hydrocortisone; MSU, monosodium monohydrate urate crystals.

marker CD163 (10). Cell culture media changes were carried out at days 1,3,5 and 7 days of culture replacing with fresh DMEM containing $10 \%$ AB serum.

Differentiated macrophages were stimulated with LPS, $(10 \mu \mathrm{g} / \mathrm{ml})$ or MSU crystals $(0.5 \mathrm{mg} / \mathrm{ml})$ with or without pre-treatment for 30 min with $\mathrm{HC}(1,2$ and $4 \mu \mathrm{M})$ Stimulation was stopped after $24 \mathrm{~h}$ of incubation in all experiments. The $0.5 \mathrm{mg} / \mathrm{ml} \mathrm{MSU}$ concentration and time point $(24 \mathrm{~h})$ was used for all experiments since this concentration and time point were previously identified as optimum conditions from previous studies of mononuclear and macrophage cell stimulation (10-13).

Macrophage stimulation. Macrophages were stimulated MSU crystals $(0.5 \mathrm{mg} / \mathrm{ml})$ or LPS $(10 \mu \mathrm{g} / \mathrm{ml})$ with or without pre-treatment for $30 \mathrm{~min}$ at $37^{\circ} \mathrm{C}$. This was followed by the addition of pathway inhibitors: AEG3482, a JNK inhibitor (12.5, 25 and $50 \mu \mathrm{M})$, MG132 a proteasome inhibitor (50 and $100 \mu \mathrm{M})$, actin D $(2 \mu \mathrm{M})$. Incubation was stopped after $24 \mathrm{~h}$ in all experiments.

Treatment with colchicine or hydrocortisone or anti-TGF $\beta 1$. Colchicine was prepared by dissolving in $1 \mathrm{ml}$ ethanol as recommended by manufacturer's instructions. Further dilutions were carried out in DMEM media at concentrations of 125,250 or $500 \mu \mathrm{g} / \mathrm{ml}$. Hydrocortisone was dissolved in $1 \mathrm{ml} \mathrm{DMEM}$ and further diluted in media to concentrations of 1,2 and $4 \mu \mathrm{M}$. Mouse anti-TGF $\beta 1$ and an isotype matched control IgG1 were both dissolved in PBS and used at 2.5, 5, $10 \mathrm{ng} / \mathrm{ml}$ (anti-TGF $\beta 1$ ) and $10 \mu \mathrm{g} / \mathrm{ml}$ (control $\mathrm{IgG1}$ ) respectively. Macrophages were pre-incubated with colchicine, hydrocortisone, anti-TGF $\beta 1$ or IgG1 control for $30 \mathrm{~min}$ prior at $37^{\circ} \mathrm{C}$ to addition of MSU crystals for $24 \mathrm{~h}$ after which supernatants were collected and analysed for PAF-AH content.

Monocyte stimulation with recombinant $P A F-A H$. Recombinant PAF-AH was dissolved in $0.5 \mathrm{ml}$ of PBS and further diluted in DMEM media (2.5-10 $\mathrm{ng} / \mathrm{ml})$. Freshly isolated monocytes were pre-treated with recombinant PAF-AH for 20 min prior to the addition of MSU crystals and then incubated at $37^{\circ} \mathrm{C}$ for $24 \mathrm{~h}$ after which supernatants were collected for cytokine analysis.

Enzyme linked immunosorbent assays (ELISAs). IL-1 $\beta$ and IL-6 concentrations in cell culture supernatants were determined by sandwich ELISA using matched antibodies (DuoSet: R\&D Systems, Abingdon, UK). PAF-AH levels were determined using a solid phase quantikine ELISA (DPLG70 from R\&D Systems) with sensitivity $(0.8-50 \mathrm{ng} / \mathrm{ml})$ in conditioned cell culture supernatants. All samples were measured in duplicates using manufacturer's instructions without any deviations. Results were expressed as the mean \pm SEM cytokine concentration $(\mathrm{ng} / \mathrm{ml})$ from at least 4 experiments.

Statistical analysis. All samples were measured in duplicate from at least three experiments, with results expressed as the mean \pm standard deviation. Statistical analysis was carried out by applying one-way analysis of variance with Dunnett's post hoc test or Student's t-test where appropriate. $\mathrm{P}<0.05$ was considered to indicate a statistically significant difference. All data were analysed using Excel (2016 version; Microsoft Corporation, Redmond, WA, USA).

\section{Results}

PAF-AH secretion by MSU crystal stimulated macrophages. MSU crystal stimulation resulted in the upregulation of PAF-AH secretion by in vitro differentiated macrophages compared to unstimulated macrophages. Peak enzyme release was detected at an incubation time of 24-48 h which was approximately six-fold higher than unstimulated macrophages (mean $\pm \mathrm{SD} ; 29.3 \pm 6.3 \mathrm{ng} / \mathrm{ml}$ vs. $5.36 \pm 0.3 \mathrm{ng} / \mathrm{ml}$ respectively, $\mathrm{P}<0.001)$ after which a decline in enzyme secretion occurred with levels reverting to near baseline levels after $72 \mathrm{~h}$ (Fig. 1).

Effect of hydrocortisone, colchicine, LPS, anti-TGF $\beta 1$ and pathway inhibitors on macrophage PAF-AH secretion. 
Co-incubation of macrophages with hydrocortisone $(1-4 \mu \mathrm{M})$ significantly decreased MSU crystal stimulated PAF secretion (Fig. 2A). Interestingly, LPS-stimulated macrophages also release PAF-AH after $24 \mathrm{~h}$ of LPS stimulation. However when we added equivalent concentration of HC to LPS stimulated macrophages we did not observe a significant decrease in PAF-AH secretion (Fig. 2). Also colchicine did not significantly alter the levels of PAF-AH at the doses used. In the next stage of our investigation we tested a variety of pathway inhibitors to investigate the signalling mechanisms involved in this model. Notably, the JNK inhibitor AEG3482 did not decrease MSU crystal-mediated PAF-AH secretion. In fact, PAF-AH release was enhanced following incubation with this inhibitor (Fig. 3). Inclusion of a proteasome inhibitor to the cultures significantly reduced PAF-AH release at the doses used (Fig. 4). In contrast, PAF-AH secretion was not significantly altered by the addition of cyclic AMP inhibitor (Fig. 5) implying that this mode of intracellular signalling was not involved in MSU-mediated PAF-AH secretion. The addition of actin $\mathrm{D}$ into the co-cultures resulted in complete amelioration of PAF-AH detection in this pathway as seen in Fig. 4 suggesting that MSU crystal de novo synthesis of PAF-AH is required in macrophages. Addition of neutralising anti-TGF $\beta 1$ resulted in a dose dependent reduction in PAF-AH at concentrations of $0.25,0.5$ and $1 \mu \mathrm{g} / \mathrm{ml}$ whereas an isotype matched IgG control antibody at the same concentrations had no effect on PAF-AH secretion (Fig. 5).

Treatment with recombinant $P A F-A H$ inhibits pro-inflammatory cytokine secretion by immature monocytes stimulated with MSU crystals. We investigated whether the activity of recombinant PAF-AH could also modify IL-1 $\beta$ and IL-6 secretion. Previously, we had reported that recombinant PAF-AH decreases TNF $\alpha$ cytokine secretion from immature human monocytes in a similar experimental model (9).

Pre-treatment of undifferentiated, inflammatory monocytes with recombinant PAF-AH at doses of 2.5, 5 and $10 \mathrm{ng} / \mathrm{ml}$ and then MSU crystals for $24 \mathrm{~h}$ resulted in a dose dependent decrease in IL-1 $\beta$ and IL-6 secretion. The highest inhibition was achieved at $10 \mathrm{ng} / \mathrm{ml}$ recombinant PAF-AH ( $\mathrm{P}=0.02$ for IL-1 $\beta$ and $\mathrm{P}=0.003$ for IL-6 respectively, Fig. 6).

\section{Discussion}

To the best of our knowledge this is the first study to identify that macrophage uptake of MSU crystals leads to an upregulation in secretion of the enzyme PAF-AH. Little is known about the enzymes involved in the inflammation resolution phase of gout with research focussing mainly on caspase-1 activating NALP3 (23,24). PAF-AH is actually an anti-inflammatory phospholipase that is found as a plasma isoform complexed mainly with LDLs (25). Low levels of PAF-AH seem to correlate with a number of modalities. A study involving patients with acute allergy reactions reported that those patients with the lowest levels of plasma PAF-AH were at high risk of severe anaphylaxis (26). Also, mast cells derived from rat bone marrow secrete PAF-AH upon direct IgE activation indicating PAF-AH may have a potentially crucial function in pathological and physiological defence. It is postulated that the likely function of PAF-AH may be

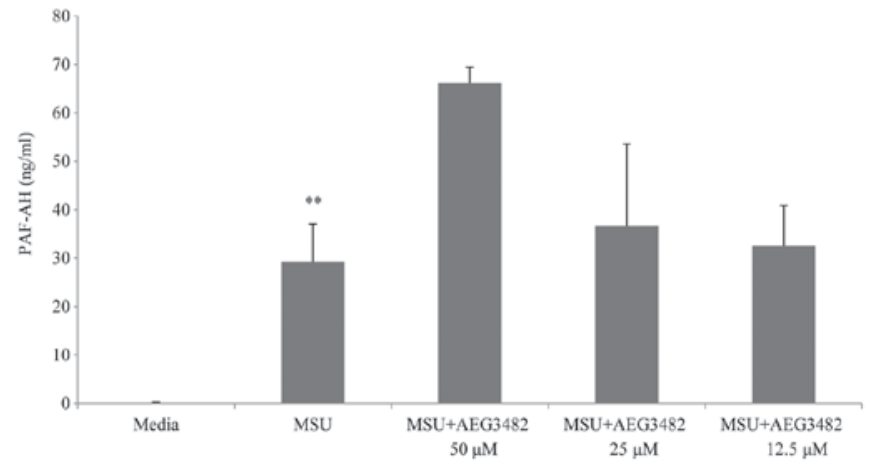

Figure 3. Effect of the c-Jun N-terminal kinase inhibitor, AEG3482, on PAF-AH release. The results revealed an upregulation of PAF-AH following the addition of the AEG3482 inhibitor to macrophages stimulated with MSU crystals in which a two-fold increase was observed by enzyme detection with $50 \mu \mathrm{M}$ AEG3482. Results are expressed as the mean \pm standard deviation of 3 similar experiments. ${ }^{* *} \mathrm{P}<0.01$ vs. Media. PAF-AH, platelet-activating factor acetyl hydrolase; MSU, monosodium monohydrate urate crystals.

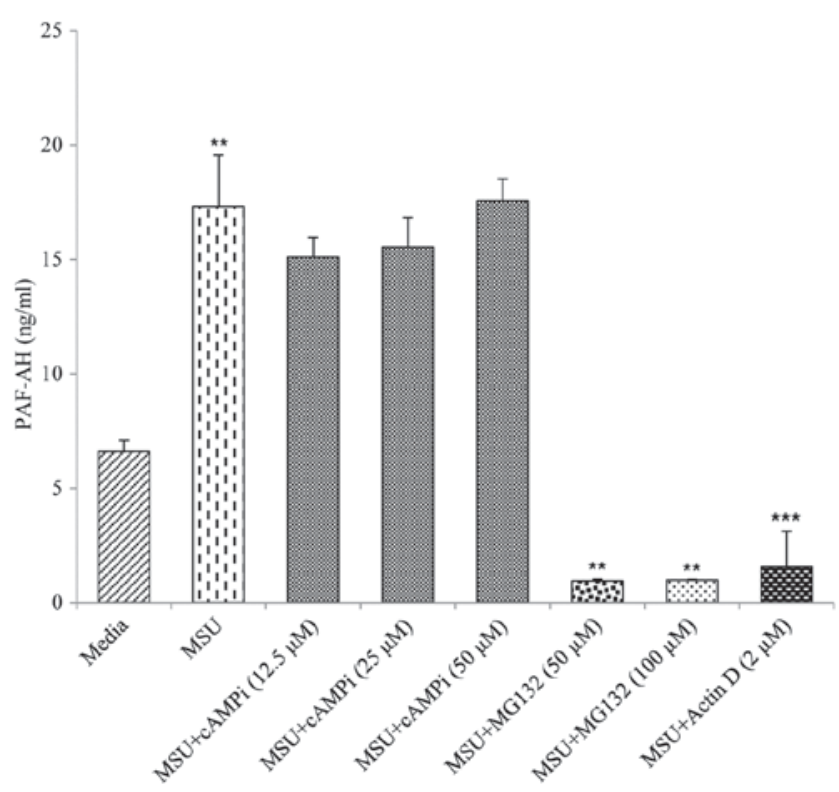

Figure 4. Effect of inhibitors on PAF-AH secretion by macrophages. Following MSU crystal stimulated macrophage pre-treatment with inhibitors downregulation of PAF-AH secretion was observed; for actin $\mathrm{D}(\mathrm{P}=0.002)$, and the proteasome inhibitor MG132 at $50 \mu \mathrm{M}(\mathrm{P}=0.08)$ and at $100 \mu \mathrm{M}$ $(\mathrm{P}=0.01)$. The inclusion of $\mathrm{cAMPi}$ at a concentration range of $12.5,25$ and $50 \mu \mathrm{M}$ did not exhibit any significant changes in PAF-AH production when compared with MSU crystal stimulated macrophages. ${ }^{* *} \mathrm{P}<0.01$ and ${ }^{* * * *} \mathrm{P}<0.001$ vs. Media. Results are expressed as the mean \pm standard expression of 3 repeated experiments. PAF-AH, platelet-activating factor acetyl hydrolase; actin D, actinomycin D; cAMPi, cyclic adenosine monophosphate inhibitor; MSU, monosodium monohydrate urate crystals.

as a safety biosensor during inflammation. This is due to its ability to neutralise the effects of inflammatory PAF and oxidised phospholipids which are upregulated in pathologies such as thrombosis, allergy, sepsis as well as gout $(26,27)$. A local increase in harmful lipids often accompanies sustained inflammation. Thus upregulation of PAF-AH takes place in cells to deactivate and degrade PAF. In fact joint inflammation in gout is accompanied by a local increase in PAF, prostaglandin E2 and leukotrienes $(28,29)$. In this study we have demonstrated that recombinant PAF-AH treatment 


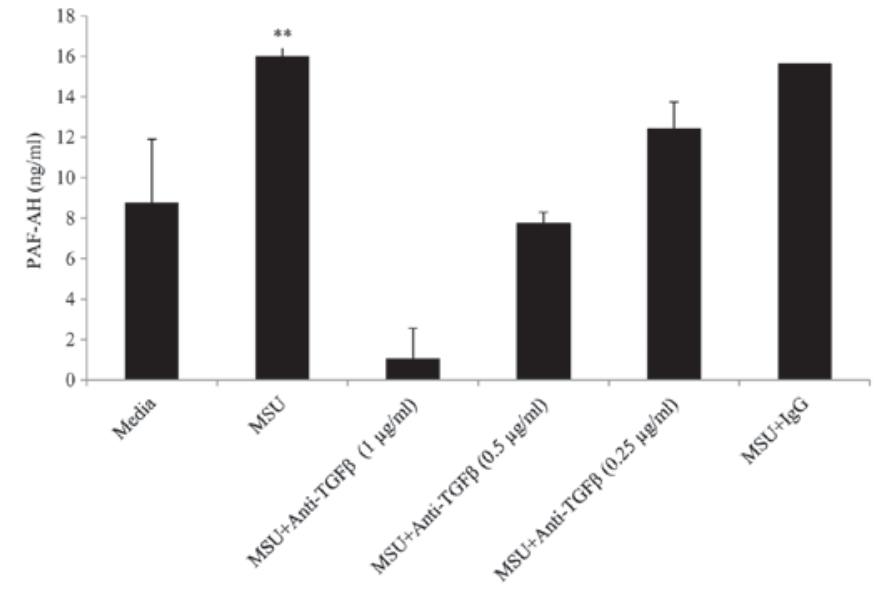

Figure 5. TGF $\beta 1$ is involved in macrophage PAF-AH secretion upon MSU crystal uptake. Differentiated macrophages were cultured in media containing a neutralising antibody to TGF- $\beta$ prior to MSU crystal stimulation for $24 \mathrm{~h}$ and the collected supernatants were analysed for PAF-AH by ELISA. Results are expressed as the mean \pm standard deviation of at least 3 experiments. ${ }^{* *} \mathrm{P}<0.01$ vs. Media. TGF $\beta 1$, transforming growth factor $\beta 1$; PAF-AH, platelet-activating factor acetyl hydrolase; IgG, immunoglobulin G; MSU, monosodium monohydrate urate crystals.

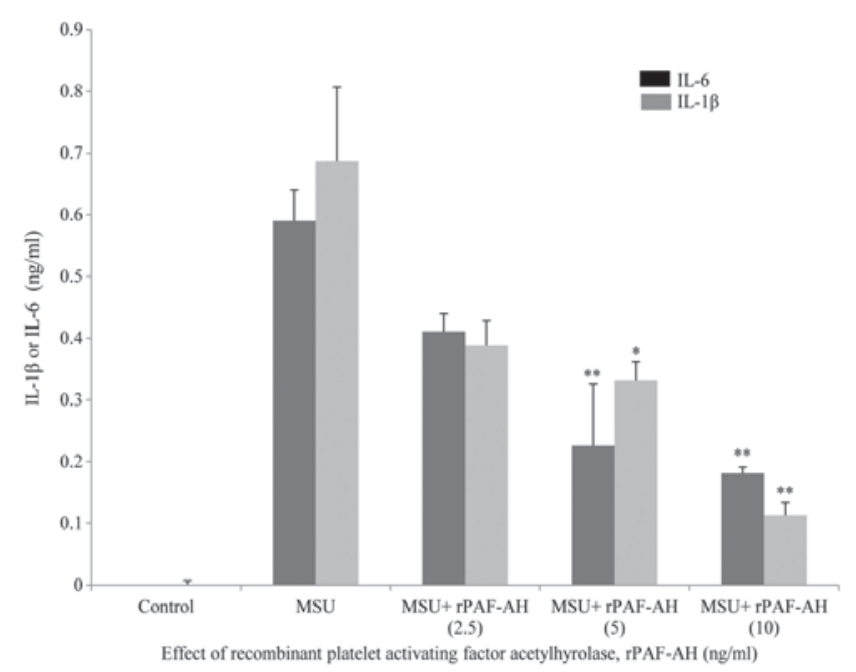

Figure 6. Downregulation of IL-1 $\beta$ and IL-6 by recombinant PAF-AH in immature monocytes stimulated with MSU crystals. Inclusion of exogenous recombinant PAF-AH resulted in a dose dependent reduction in IL- $1 \beta$ release by monocytes. Statistical significance was observed at $5 \mathrm{ng} / \mathrm{ml}$ (IL-1 $\beta, \mathrm{P}=0.03$; IL-6, P=0.003) and at $10 \mathrm{ng} / \mathrm{ml}(\mathrm{IL}-1 \beta, \mathrm{P}=0.008$; IL-6, P=0.003) with respect to recombinant PAF-AH pre-treatment of macrophage cultures. Results are expressed as the mean \pm standard deviation of at least 3 experiments. ${ }^{*} \mathrm{P}<0.05$ and ${ }^{* * *} \mathrm{P}<0.01$ vs. MSU only. rPAF-AH, recombinant platelet-activating factor acetyl hydrolase; IL, interleukin; MSU, monosodium monohydrate urate crystals.

may tip the inflammatory balance in favour of inflammation resolution as it was able to decrease monocyte derived IL- $1 \beta$ and IL-6 secretion due to MSU crystal stimulation. These results are consistent with our previous study in which PAF was upregulated by monocytes in response to MSU crystals (9) and recombinant PAF-AH administration downregulated TNF $\alpha$ secretion. The exact form of PAF was not characterised; we propose that this was probably inert lysosomal PAF. Physiologically PAF-AH functions on active PAF, converting it into lysosomal-PAF (the inert form of PAF). Active PAF is a potent lipid intermediate that has immunomodulatory effects and a pivotal role in the pathogenesis of inflammatory disorders such as cardiovascular disease rendering this molecule inactive is important in healing (30). Given this function, it is not surprising that administration of exogenous PAF-AH to animals with systemic inflammatory response syndromes or sepsis resulted in decreased leukocyte accumulation as well as pro-inflammatory cytokine levels and increased bacterial clearance in septic mice. Indeed PAF-AH enhances sepsis clearance by hydrolysing acetyl groups attached to other lipid substrates besides PAF $(31,32)$. This function of the enzyme in neutralising inflammatory mediators could be particularly crucial in inflammatory gout joints in which a number of lipid mediators such as Prostaglandin E2 and PAF have been located (28). In endotoxaemic rats, an upregulation of plasma PAF-AH was accompanied by a direct increase in ability to inactivate PAF and oxidised phospholipids (33).

We discovered that blocking TGF $\beta 1$ activity using a neutralising antibody resulted in a dose dependant decrease in PAF-AH secretion. The anti-inflammatory activity of TGF $\beta 1$ is well established in gout inflammation resolution (13). Our results suggest that this effect may be attributed in part by the upregulation of PAF-AH. Essentially both PAF-AH and TGF $\beta 1$ share a transcription binding site involved in macrophage regulation, mediating effects through canonical Sp1 $(16,34)$. Hydrocortisone is known for its anti-inflammatory properties through suppression of vascular permeability, vasodilation and leukocyte migration into inflamed sites (35). Unexpectedly in our study hydrocortisone decreased PAF-AH secretion by MSU crystal stimulated macrophages. However, there is evidence that hydrocortisone may also have pro-inflammatory properties. For example, hydrocortisone can interact with anti-inflammatory drugs such as aspirin hydrolysis where it mediates esterase activity (36). Furthermore, when normal subjects were injected with $300 \mathrm{mg}$ of hydrocortisone, there was an increase in toll like inflammatory receptor 2, 5, 9, high mobility group box-1 and matrix metalloproteinase- 9 expression occurred (37). Perhaps the long term use of hydrocortisone therapy in gout or arthritis could result in inadvertently driving the inflammatory response rather than limiting it.

Co-incubation of MSU-stimulated macrophages with the proteasome inhibitor MG132 resulted in a significant decrease in PAF-AH secretion. This suggests that this pathway involves protein degradation (38). Conversely, colchicine had no significant effect. Colchicine is routinely prescribed as a prophylactic treatment for gout at similar doses tested in our study. It has recognized, powerful anti-inflammatory properties in gout affecting cytokine levels; increasing levels of TGF $\beta 1$, prostaglandins and lowering pro-inflammatory factor levels (39).

Our results could be relevant to other arthropathic disorders such as rheumatoid arthritis (RA) as well as gout. Recently, it has been shown that PAF-AH levels are significantly decreased in the sera of RA patients with active disease highlighting that PAF-AH probably plays a protective role in the development of RA (40). Moreover analysis of the hypercoagulable state in RA patients showed significant reduction in anti-inflammatory serum PAF-AH along with a decrease in IL- 4 and IL-10 but a rise in inflammatory mediators such as IL-6, IL-17 and PAF (41). Therefore future studies should examine the expression profile of PAF-AH levels in 
the synovial fluid samples obtained from gout patients during active disease compared to quiescent joints. The efficacy of PAF-AH treatment for gout patients should also be explored. In conclusion, we have demonstrated that PAF-AH enzyme secretion is upregulated by macrophages following MSU crystal uptake and involves anti-inflammatory TGF $\beta 1$. This research expands our understanding of an important anti-inflammatory enzyme which could be functioning as a biosensor responding to local microenvironmental conditions.

\section{Acknowledgements}

Not applicable.

\section{Funding}

The present study was funded by the Middlesex University Department of Natural Sciences Research Initiative (2014).

\section{Availability of data and materials}

The datasets used and/or analyzed during the current study are available from the corresponding author on reasonable request.

\section{Authors' contributions}

DY designed all of the experiments, collected and analyzed the data, performed statistical analysis and wrote the manuscript. FH contributed to data analysis and writing the manuscript.

\section{Ethics approval and consent to participate}

The present study incorporated a series of in vitro cell cultures of differentiated monocytes isolated from human blood cones purchased from the National Health Service Blood Bank (Colindale, London, England). Ethical approval was granted by the Middlesex University Institutional Ethics Committee, Department of Natural Sciences (London, England) and this was also a purchase requirement from the blood bank. The present study complied with the ethical standards established in 1964 in the Declaration of Helsinki.

\section{Patient consent for publication}

Not applicable.

\section{Competing interests}

The authors declare that they have no competing interests.

\section{References}

1. Hippocrates: The Genuine Works of Hippocrates. Volume I and II. Adams F (ed). New York: William Wood and Company, 1886

2. Copeman WS: A short history of the gout and the rheumatic diseases. Berkeley and Los Angeles. University of California Press, 1964.

3. Kuo CF, Grainge MJ, Mallen C, Zhang W and Doherty M: Rising burden of gout in the UK but continuing suboptimal management: A nationwide population study. Ann Rheum Dis 72: 661-667, 2015
4. di Giovine FS, Malawista SE, Thornton E and Duff GW: Urate crystals stimulate production of tumour necrosis factor alpha from human blood monocytes and synovial cells. Cytokine mRNA and protein kinetics, and cellular distribution. J Clin Invest 87: 1375-1381, 1991.

5. Schiltz C, Lioté F, Prudhommeaux F, Meunier A, Champy R, Callebert J and Bardin T: Monosodium urate monohydrate crystal induced inflammation in vivo: Quantitative histomorphometric analysis of cellular events. Arthritis Rheum 46: 1643-1650, 2002.

6. Daoussi D, Andonpoulos I and Andonopoulos AP: ACTH as a treatment for acute crystal induced arthritis: Update on clinical evidence and mechanisms of action. Semin Arthritis Rheum 43: 648-653, 2014.

7. Vanja SK, Rathinam VA and Fitzgerald KA: Mechanisms of inflammasome activation: Recent advances and novel insights. Trends Cell Biol 25: 308-315, 2015.

8. Yagnik DR, Hillyer P, Marshall D, Smythe CD, Krausz T, Haskard DO and Landis RC: Noninflammatory phagocytosis of monosodium urate monohydrate crystals by mouse macrophages. Implications for the control of joint inflammation in gout. Arthritis Rheum 43: 1779-1789, 2000.

9. Yagnik D: Macrophage derived platelet activating factor implicated in the resolution phase of gouty inflammation. Int $\mathrm{J}$ Inflam 2014: 526496, 2014.

10. Landis RC, Yagnik DR, Florey O, Philippidis P, Emons V, Mason JC and Haskard DO: Safe disposal of inflammatory monosodium urate monohydrate crystals by differentiated macrophages. Arthritis Rheum 46: 3026-3033, 2002.

11. Yagnik DR, Evans BJ, Florey O, Mason JC, Landis RC and Haskard DO: Macrophage release of transforming growth factor betal during resolution of monosodium urate monohydrate crystal-induced inflammation. Arthritis Rheum 50: 2273-2280, 2004.

12. Howard KM, Abdel-Al M, Ditmyer $\mathrm{M}$ and Patel $\mathrm{N}$ : Lipopolysaccharide and platelet-activating factor stimulate expression of platelet-activating factor acetylhydrolase via distinct signaling pathways. Inflam Res 60: 735-744, 2011.

13. Richette P, Doherty M, Pascual E, Barskova V, Becce F, Castañeda-Sanabria J, Coyfish M, Guillo S, Jansen TL, Janssens H, et al: 2016 updated EULAR evidence-based recommendations for the management of gout. Ann Rheum Dis 76: 29-42, 2017.

14. Elstad MR, Stafforini DM, McIntyre TM, Prescott SM and Zimmerman GA: Platelet-activating factor acetylhydrolase increases during macrophage differentiation. A novel mechanism that regulates accumulation of platelet-activating factor. J Biol Chem 25: 8467-8470, 1989.

15. Narahara H and Johnston JM: Effects of endotoxins and cytokines on the secretion of platelet-activating factor-acetylhydrolase by human decidual macrophages. Am J Obstet Gynecol 169: 531-537, 1993.

16. Wu X, Zimmerman GA, Prescott SM and Stafforini DM: The p38 MAPK pathway mediates transcriptional activation of the plasma platelet-activating factor acetylhydrolase gene in macrophages stimulated with lipopolysaccharide. J Biol Chem 279: 36158-36165, 2004.

17. Hattori $\mathrm{K}$, Hattori $\mathrm{M}$, Adachi $\mathrm{H}$, Tsujimoto $\mathrm{M}$, Arai $\mathrm{H}$ and Inoue K: Purification and characterization of platelet-activating factor acetylhydrolase II from bovine liver cytosol. J Biol Chem 270: 22308-22313, 1995.

18. Stafforini DM, McIntyre TM, Zimmerman GA and Prescott SM: Platelet-activating factor acetylhydrolases. J BiolChem 272: 17895-17898, 1997.

19. Stafforini DM, Prescott SM, Zimmerman GA and McIntyre TM: Mammalian platelet-activating factor acetylhydrolases. Biochim Biophys Acta 1301: 161-173, 1996.

20. Stafforini DM, Prescott SM and McIntyre TM: Human plasma platelet-activating factor acetylhydrolase. Purification and properties. J Biol Chem 262: 4223-4230, 1987.

21. Castro Faria Neto HC, Stafforini DM, Prescott SM and Zimmerman GA: Regulating inflammation through the anti-inflammatory enzyme platelet-activating factor-acetylhydrolase. Mem Inst Oswaldo Cruz 100 (Suppl 1): S83-S91, 2005.

22. Blank ML, Lee T, Fitzgerald V and Snyder F: A specific acetylhydrolase for 1-alkyl-2-acetyl-sn-glycero-3-phosphocholine (a hypotensive and platelet-activating lipid). J Biol Chem 256: 175-178, 1981.

23. Akira S, Misawa T, Satoh T and Saitoh T: Macrophages control innate inflammation. Diabetes Obes Metab 15 (Suppl 3): S10-S18, 2013. 
24. So AK and Martinon F: Inflammation in gout: Mechanisms and therapeutic targets. Nat Rev Rheumatol 13: 639-647, 2017.

25. Triggiani M, Granata F, Giannattasio G and Marone G: Secretory phospholipases A2 in inflammatory and allergic diseases: Not just enzymes. J Allergy Clin Immunol 116: 1000-1006, 2005

26. Perelman B, Adil A and Vadas P: Relationship between platelet activating factor acetylhydrolase activity and apolipoprotein B levels in patients with peanut allergy. Allergy Asthma Clin Immunol 10: 20, 2014.

27. Nakajima K, Murakami M, Yanoshita R, Samejima Y, Karasawa K, Setaka M, Nojima S and Kudo I: Activated mast cells release extracellular type platelet-activating factor acetylhydrolase that contributes to autocrine inactivation of platelet-activating factor. J Biol Chem 272: 19708-19713, 1997.

28. Vadas P, Browning J, Edelson J and Pruzanski W: Extracellular phospholipase A2 expression and inflammation: The relationship with associated disease states. J Lipid Mediat 8: 1-30, 1993.

29. MiguélezR,PalaciosI,NavarroF,GutierrezS,Sanchez-PernauteO, Egido J, González E and Herrero-Beaumont G: Anti-inflammatory effect of a PAF receptor antagonist and a new molecule with antiproteinase activity in an experimental model of acute urate crystal arthritis. J Lipid Mediat Cell Signal 13: 35-49, 1996.

30. Tjolker LW, Eberhardt C, Unger J, Trong HL, Zimmerman GA, McIntyre TM, Stafforini DM, Prescott SM and Gray PW: Plasma platelet activating factor acetylhydrolase is a secreted phospholipase A2 with a catalytic triad. J Biol Chem 270: 25481-25487, 1995.

31. Gomes RN, Bozza FA, Amâncio RT, Japiassú AM, Vianna RC, Larangeira AP, Gouvêa JM, Bastos MS, Zimmerman GA, Stafforini DM, et al: Exogenous platelet activating factor acetylhyhrolase reduces mortality in mice with systemic inflammatory response syndrome and sepsis. Shock 26: 41-49, 2006.

32. Opal S, Laterre PF, Abraham E, Francois B, Wittebole X, Lowry S, Dhainaut JF, Warren B, Dugernier T, Lopez A, et al: Recombinant human platelet-activating factor acetylhydrolase for treatment of severe sepsis: Results of a phase III, multicenter, randomized, double-blind, placebo-controlled, clinical trial. Crit Care Med 32: 332-341, 2004
33. Howard KM and Olson MS: The expression and localization of plasma platelet-activating factor acetylhydrolase in endotoxemic rats. J Biol Chem 275: 19891-19896, 2000.

34. Li JM, Datto MB, Shen X, Hu PP, Yu Y and Wang XF: Sp1, but not $\mathrm{Sp} 3$, functions to mediate promoter activation by TGF-beta through canonical Sp1 binding sites. Nucleic Acids Res 26: 2449-2456, 1998

35. Coutinho AE and Chapman KE: The anti-inflammatory and immunosuppressive effects of glucocorticoids, recent developments and mechanistic insights. Mol Cell Endocrinol 335: 2-13, 2011.

36. Zhou G, Marathe GK, Hartiala J, Hazen SL, Allayee H, Tang WH and McIntyre TM: Aspirin hydrolysis in plasma is a variable function of butyrylcholinesterase and platelet-activating factor acetylhydrolase 1b2 (PAFAH1b2). J Biol Chem 288: 11940-11948, 2013.

37. Dandona P, Ghanim H, Sia CL, Green K, Abuaysheh S, Dhindsa S, Chaudhuri A and Makdissi A: A mixed anti-inflammatory and pro-inflammatory response associated with a high dose of corticosteroids. Curr Mol Med 14: 793-801, 2014.

38. Guo N and Peng Z: Mg132, a proteasome inhibitor, induces apoptosis in tumour cells. Asia Pac J Clin Oncol 9: 6-11, 2013.

39. Dalbeth N, Lauterio TJ and Wolfe HR: Mechanism of action of colchicine in the treatment of gout. Clin Ther 36: 1465-1479, 2014.

40. Łuczaj W, Gindzienska-Sieskiewicz E, Jaroka-karpowicz I, Andisic L, Sierakowski S, Zarkovic N, Waeg G and Skrzydlewska E: The onset of lipid peroxidation in rheumatoid arthritis: Consequences and monitoring. Free Radic Res 50: 304-313, 2016.

41. Zhang P, Liu J, Tan B, Zhu F and Fang L: Hypercoagulable state is associated with NF-kappa B activation and increased inflammatory factors in patients with rheumatoid arthritis. Xi Bao Yu Fen Zi Mian Yi Xue Za Zhi 32: 364-368, 2016 (In Chinese). 